\title{
Bilateral Simultaneous Anterior Ischemic Optic Neuropathy Associated with Sildenafil
}

\author{
Marilita M. Moschos ${ }^{a}$ loannis Margetis ${ }^{b}$ \\ aDepartment of Ophthalmology, University of Athens, and \\ 'Department of Ophthalmology, 'Evgenidio' Hospital, Athens, Greece
}

\section{Key Words}

Nonarteritic anterior ischemic optic neuropathy $\cdot$ Sildenafil $\cdot$ Visual acuity $\cdot$ Visual fields

\begin{abstract}
We report a case of simultaneous bilateral nonarteritic anterior ischemic optic neuropathy (NAION) due to sildenafil use. A 55-year-old man with an unremarkable medical history presented simultaneous bilateral NAION 8 months after continuous use of sildenafil 4-5 times a month. At presentation, visual acuity (VA) was 0.7 in the right eye (RE) and 0.9 in the left eye (LE). The visual field showed an inferior altitudinal defect in both eyes and a fundus examination revealed prominent optic disc edema in the RE and a crowded optic disc in the LE. The patient was counseled to discontinue sildenafil, and 3 weeks later VA was 1.0 in both eyes and the optic disc edema in the RE was resolved. However, a visual field defect remained in the RE. Three months later, visual fields were unchanged. To the best of our knowledge, this is the first reported case of simultaneous bilateral NAION due to sildenafil use.
\end{abstract}

\section{Introduction}

Sildenafil citrate (Viagra) is a potent and selective cyclic guanosine monophosphate (cGMP)-specific phosphodiesterase type 5 (PDE5) inhibitor. Increased cGMP results in relaxation and vasodilation of vascular smooth muscle $[1,2]$ and enhances penile erectile function in men. Some reports suggest that sildenafil use may be associated with nonarteritic anterior ischemic optic neuropathy (NAION) [3, 4], and several patients have developed NAION between 1 and $12 \mathrm{~h}$ after taking sildenafil [5]. We report, for the first time, to our knowledge, a case of bilateral simultaneous anterior NAION after taking sildenafil. 


\section{Case Report}

A 55-year-old man with an unremarkable medical history had noticed vision troubles in both eyes, associated with nausea and headache. Over the previous 8 months, he had taken $50 \mathrm{mg}$ of sildenafil 4-5 times a month. At presentation, visual acuity (VA) was 0.7 in the right eye (RE) and 0.9 in the left eye (LE), with a right-sided relative afferent pupillary defect. The patient was able to read Ishihara plates with each eye separately and without any errors; testing with a Humphrey Visual Field Analyzer confirmed an inferior altitudinal defect in both the RE and LE. Fundus examination revealed prominent optic disc edema with some rim disc hemorrhages in the RE. In the LE, the optic disc was crowded with a blurred optic disc margin and distended retinal veins. Blood pressure and pulse measured 130/60 $\mathrm{mm}$ $\mathrm{Hg}$ and 90 beats per minute, respectively. Laboratory testing revealed a normal antinuclear antibody titer, an erythrocyte sedimentation rate of $10 \mathrm{~mm} / \mathrm{h}$, a normal complete blood count, and a normal tuberculosis skin test. Also, an MRI of the brain with gadolinium and fat suppression demonstrated nothing abnormal. The absence of any evidence of a systemic inflammatory disease led us to conclude that the patient had experienced bilateral NAION mainly in the RE. The patient was counseled to discontinue sildenafil without any other treatment. Three weeks later, VA was 1.0 in both eyes; the visual field in the LE was normal, but the visual field defect in the RE remained. Optic disc edema in the $\mathrm{RE}$ was resolved. Three months after presentation, visual fields were unchanged (fig. 1).

\section{Discussion}

Since 2001, sporadic cases of NAION due to sildenafil use have been reported [3-5]. In all these cases, the affection was unilateral and most had a small, crowded optic nerve head ('disc at risk'). Only 2 cases of bilateral NAION have been reported to date. However, in the first of these cases, the patient had experienced a previous episode in the fellow eye several months before starting treatment with sildenafil [3]. According to the authors, the patient had bilateral disc-at-risk appearance. In the second case, the patient experienced bilateral posterior NAION after a megadose of sildenafil; he also had a history of systemic hypertension, lipidemia and stroke [6]. To the best of our knowledge, ours is the first reported case of bilateral simultaneous NAION in a patient without prior medical history or other ischemic stigmata. We suggest, like others [7], that the disc-atrisk configuration predisposes patients to NAION, and it is reasonable to advise these patients to be cautious about using sildenafil or any other medication that might cause systematic hypotension.

\section{Disclosure Statement}

The authors have no financial support or proprietary interests to declare in relation to this article. 

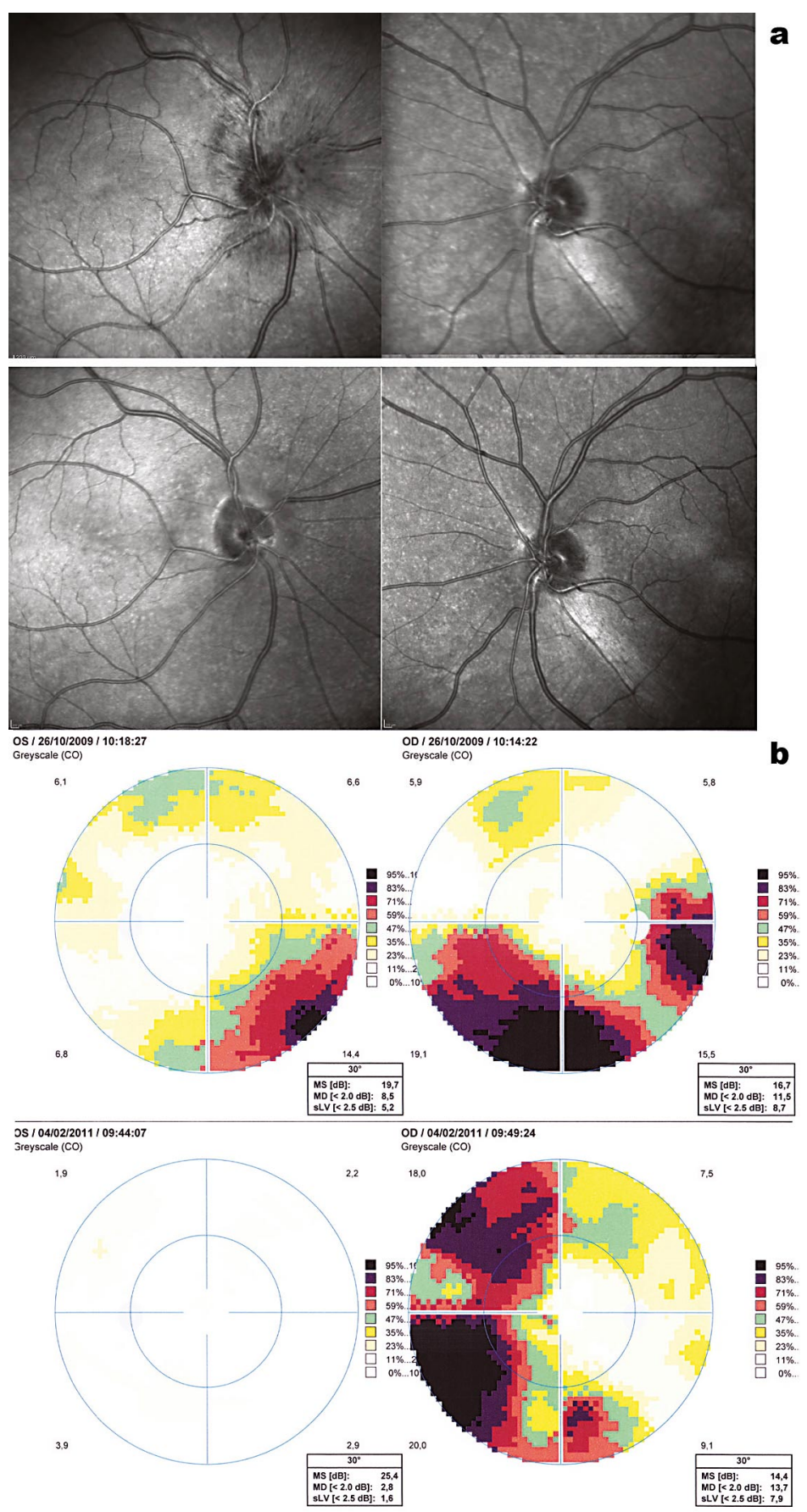

Fig. 1. a Appearance of both optic discs at presentation and 3 months afterward. b Visual field defects at presentation and 3 months afterward. 


\section{References}

1 Ballard SA, Gingell CJ, Tang K, Turner LA, Price ME, Naylor AM: Effects of sildenafil on the relaxation of human corpus cavernosum tissue in vitro and on the activities of cyclic nucleotide phosphodiesterase isozymes. J Urol 1998;159:2164-2171.

2 Zussman RM, Morales A, Glasser DB, Osterloh IT: Overall cardiovascular profile of sildenafil citrate. Am J Cardiol 1999;83:35C-44C.

-3 Cunningham AV, Smith KH: Anterior ischemic optic neuropathy associated with viagra. J Neuroophthalmol 2001;21:22-25.

4 Gruhn N, Fledelius AC: Unilateral optic neuropathy with sildenafil intake. Acta Ophthalmologica 2005;83:121-132.

5 El-Domyati MM, El-Fakahany HM, Morad KE: Nonarteritic ischaemic optic neuropathy (NAION) after $36 \mathrm{~h}$ of intake of sildenafil citrate: first Egyptian case. Andrologia 2009;41:319-321.

-6 Su DH, Ang PS, Tow SL: Bilateral posterior ischemic optic neuropathy associated with use of sildenafil. J Neuroophthalmol 2008;28:75.

7 Pomeranz HD, Smith KH, Hart WM Jr, Ergan RA: Sildenafil associated nonarteritic anterior ischemic optic neuropathy. Ophthalmology 2002;109:584-587. 